\title{
Surface electrical stimulation of skeletal muscle after spinal cord injury
}

\author{
EA Hillegass ${ }^{1}$ and GA Dudley*,2 \\ ${ }^{1}$ Department of Physical Therapy, Georgia State University, Atlanta, Georgia 30303, USA and ${ }^{2}$ Department of \\ Exercise Science, The University of Georgia, Athens, Georgia 30602, USA
}

Study design: Survey.

Objective: Examine muscle contractile activity during electrical stimulation (ES) after spinal cord injury (SCI).

Setting: General community of Athens, Georgia, USA.

Methods: Eight clinically complete SCI adults (C6 to T12) $4 \pm 1$ (mean \pm SE) years post injury and eight able-bodied adults were studied. Surface ES was applied to the left $\mathrm{m}$. quadriceps femoris for three sets of $10,1 \mathrm{~s}$ isometric actions $(50 \mathrm{~Hz}$ trains, $400 \mu \mathrm{s}$ biphasic pulses, $50 \mu$ s phase delay, $1 \mathrm{~s}: 1 \mathrm{~s}$ duty cycle) with $90 \mathrm{~s}$ of rest between sets. Current was set to evoke isometric torque that was (1) sufficient to elicit knee extension with $2.3 \mathrm{~kg}$ attached to the ankle (low level ES), and (2) intended to equal 30\% (mid level ES) or $60 \%$ of maximal voluntary torque of able-bodied adults (high level ES, able-bodied only). The absolute and relative cross-sectional area (CSA) of $m$. quadriceps femoris that was stimulated as reflected by contrast shift in magnetic resonance images and torque were measured.

Results: Six $\pm 2,20 \pm 2$ and $38 \pm 4 \%$ of the average CSA of $m$. quadriceps was stimulated during low, mid and high level ES, respectively, for able-bodied. Corresponding values for SCI for low and mid level ES were greater $(61 \pm 12$ and $92 \pm 7 \%, P=0.0002)$. Torque was related to the CSA $\left(\mathrm{cm}^{2}\right)$ of stimulated muscle $\left(\mathrm{Nm}=3.53 \times\right.$ stimulated CSA $+13, r^{2}=0.68$, $P=0.0010)$, thus ES of a greater per cent of $m$. quadriceps femoris in SCI was attributed to their smaller muscle $\left(24 \pm 3\right.$ vs $\left.73 \pm 5 \mathrm{~cm}^{2}, P=0.0001\right)$. The decline in torque ranged from $9 \pm 1$ to $15 \pm 4 \%$ within and over sets for low, mid or high level ES in able-bodied. SCI showed greater $(P=0.0001)$ fatigue $(19 \pm 3$ to $47 \pm 6 \%)$.

Conclusion: The territory of muscle activation by surface electrical stimulation varies among SCI patients. Given sufficient current, a large portion of the muscle of interest can be stimulated. The resulting torque is modest, however, compared to that attainable in ablebodied individuals due to the small size and limited fatigue resistance of skeletal muscle years after spinal cord injury.

Keywords: spinal cord injury; skeletal muscle; magnetic resonance imaging; electrical stimulation

\section{Introduction}

Muscle wasting and conversion to a faster muscle with low aerobic-oxidative enzyme content are components of the neuromuscular sequela to spinal cord injury (SCI) ${ }^{1-9}$ As a result of these adaptations, resistance to fatigue and force development of affected skeletal muscle are compromised. For example, $m$. quadriceps femoris, tibialis anterior or soleus show loss of force during repeat contractions evoked by surface electrical stimulation (ES) that increases with time after injury or is greater than that presented by able-bodied controls. ${ }^{10-14}$ Less consistent are findings concerning force development. Surface ES a year or longer after complete SCI has been shown to evoke isometric forces that are $1 / 7$ to $1 / 3$ of those of able-bodied controls. ${ }^{10-12} \mathrm{In}$

*Correspondence: GA Dudley, PhD, Department of Exercise Science, 115 F Ramsey Center, The University of Georgia, 300 River Road, Athens, Georgia 30602, USA contrast, force evoked by ES of $m$. tibialis anterior in SCI patients has been reported to be comparable to that of able-bodied controls. ${ }^{13}$ As the authors acknowledged, ${ }^{13}$ it is difficult to activate all fibers within a muscle, especially where pain could limit ES amplitude in able-bodied controls. Nevertheless, Shields ${ }^{14}$ has also suggested that torque during $\mathrm{ES}$ of $\mathrm{m}$. soleus is not compromised in patients a year or longer after SCI.

These apparent inconsistencies illustrate the difficulty in determining the extent of skeletal muscle activation by ES. Magnetic resonance imaging (MRI) offers the opportunity to study activation of muscle in vivo. ${ }^{15-21}$ An increase in the time constant of decay of the MR signal $\left(\mathrm{T}_{2}\right)$ has been associated with previous contractile activity. The extent of $T_{2}$ contrast shift of muscle is correlated with previous contractile activity. The extent of $\mathrm{T}_{2}$ contrast shift of muscle is correlated with integrated electromyography activity ${ }^{15}$ and 
increases with exercise intensity. ${ }^{15,16,19,22-24}$ Adams et $a l^{25}$ utilized contrast shifts in MR images to reflect the territory and extent of muscle contraction induced by surface ES. The results showed that the location and territory of muscle stimulation cannot be assumed to be identical across subjects. In addition, a linear relation between the CSA of the $m$. quadriceps femoris that was stimulated and the resultant torque was demonstrated. Accordingly, the purpose of this study was to examine the extent of stimulation of skeletal muscle by ES in SCI patients and able-bodied adults. This also afforded the opportunity to examine the territory of stimulation and compare force and fatigue during repeat sets of ES between these groups.

\section{Methods}

\section{General design}

Subjects were recruited from discharge records of the Shepherd Center, Atlanta, GA (SCI adults) and from the faculty, staff and student population of The University of Georgia (able-bodied adults). Both groups had MR images taken of both thighs at rest, before ES, and immediately after each of two (SCI) or three (able-bodied) levels of ES of increasing current. Each level consisted of three sets of ten $1 \mathrm{~s}$ isometric actions. Isometric torque for low and mid level ES was intended to be comparable between groups. For low level ES, current was set to that which evoked knee extension with $2.3 \mathrm{~kg}$ attached to the ankle. This is a standard practice in functional electrical stimulation protocols. ${ }^{26}$ Subsequently, this current was used to induce isometric contractions. Current was increased for mid level ES and was intended to evoke an isometric torque equal to $30 \%$ of maximal voluntary isometric torque for able-bodied adults. High level ES for able-bodied adults only was done to provide a wide range of values for the CSA of stimulated muscle and the resultant isometric torque. For this, current amplitude was further increased to evoke torque equal to $60 \%$ of maximal voluntary isometric torque. Isometric torque arising from ES and the accompanying contraction-incuded contrast shift were subsequently assessed.

\section{Subjects}

Eight SCI adults (six male and two females) between 18-45 years of age that lived within commuting distance to Athens, GA, USA, were recruited to participate. All were identified as clinically complete motor lesions, demonstrated a range of motion about the hip and knee joints to be within functional limits, and were free of any lower limb orthopaedic deformities. Each was rated for spasticity per the modified Ashworth Scale (Scored 0-3). In addition, seven males and one female between the ages of $18-45$ years who had not suffered any injury to their spinal cord were recruited to participate as able-bodied adults. Before any tests were conducted, the protocol and tests to be used in this study and the potential risks and benefits of participation were explained to each subject who subsequently signed an informed consent approved by the Institutional Review Board at The University of Georgia.

\section{Electrical stimulation}

Surface ES of the left $m$. quadriceps femoris was done using a commercially available stimulator (TheraTouch model 4.7, Rich-Mar Corporation, Inola, OK, USA) and laboratory designed dynamometer. The dynamometer consisted of a chair with a rigid lever arm positioned $70^{\circ}$ below horizontal. A moment arm of one foot was established by mounting a load cell (model RL 20000A, Rice Lake Weighing Systems, Rice Lake, WI, USA) perpendicular to and 12 inches from the axis of rotation of the lever arm. Isometric torque in footpounds was recorded on a chart recorder (model 2400S, Gould Instrument Systems, Valley View, OH, USA), and subsequently converted to $\mathrm{Nm}$. The dynamometer was calibrated by hanging known weights on the load cell.

Each able-bodied subject performed three maximal voluntary isometric contractions with $1 \mathrm{~min}$ of rest between efforts, as done previously. ${ }^{20,21,25}$ This was done to determine the isometric torque ES would need to elicit to equal $30 \%$ and $60 \%$ of maximal voluntary. Next, the current that was sufficient to elicit full knee extension during a dynamic contraction was determined. Briefly, a $2.3 \mathrm{~kg}$ cuff was attached to each subject's left calf juxtapose to the ankle (SCI and ablebodied) and two 7.5 by $10 \mathrm{~cm}$ oval reusable electrodes (Excel Plus, AR Davis Company, Irvington, AL, USA) were placed over $m$. quadriceps femoris; one $9 \mathrm{~cm}$ above the superior aspect of the patella and the other lateral to and $26 \mathrm{~cm}$ above the patella over $m$. vastus lateralis. Subjects then received ES $(50 \mathrm{~Hz}$ train of $400 \mu \mathrm{s}$ biphasic pulse, $50 \mu \mathrm{s}$ phase delay). With the subject seated, knee joint flexed $90^{\circ}$ and the left leg suspended, ES current was gradually increased from $0 \mathrm{mAmp}$ to that which evoked full knee extension. This took about $2 \mathrm{~s}$. The current at full knee extension was recorded. The $2.3 \mathrm{~kg}$ was removed, the leg was attached to the rigid lever arm of the dynamometer and the bouts of isometric actions were conducted.

Each subject received ES with the current that induced weighted knee extension for low level ES. Current was increased for each able bodied subject to evoke a torque that was equal to $30 \%$ and $60 \%$, respectively, of maximal voluntary isometric torque for mid and high level ES. The intent of mid level ES for SCI subjects was to increase current sufficiently to evoke a torque that was comparable to the average torque developed by the able-bodied subject for mid level ES. Able-bodied subjects rested $45 \mathrm{~min}$ between levels of ES to allow recovery. SCI subjects showed little recovery after low level ES, thus their mid level ES was conducted 2 to 4 weeks later. Three sets of ten 
contractions $(1 \mathrm{~s} 50 \mathrm{~Hz}$ train of $400 \mu$ s biphasic pulse, $50 \mu$ s phase delay) with $1 \mathrm{~s}$ of rest between actions and $90 \mathrm{~s}$ between sets were evoked for each level of ES, essentially as done previously. ${ }^{25}$ This frequency and pulse duration were used to evoke maximal tetanic force $^{31}$ and thereby a marked metabolic demand of the activated skeletal muscle. Consequently, stimulated skeletal muscle would be expected to show measurable contrast shift. ${ }^{25}$

\section{Magnetic resonance imaging (MRI)}

Standard spin-echo MR images of the thigh were collected using a 1.5 Tesla super conducting magnet (General Electric, Milwaukee, WI, USA). Contiguous trans-axial $1 \mathrm{~cm}$ thick images $(\mathrm{TR} / \mathrm{TE}=2000 / 30,60$; $\mathrm{FOV}=40 \mathrm{~cm}$ ) spaced $1 \mathrm{~cm}$ apart were collected from the head of the femur to the knee joint, essentially as done previously. ${ }^{20,21,25,27,28}$ A $256 \times 128$ matrix was acquired with one excitation. This image acquisition protocol was used to provide high spatial resolution in a reasonable time (under $6 \mathrm{~min}$ ) and to allow direct comparison with studies utilizing similar two echo collections.

Digitalized MR images were transferred to a computer for calculation of muscle cross-sectional area (CSA) and $T_{2}$ using a modified version of the public domain NIH Image program (written by Wayne Rasband at the U.S. National Institutes of Health, and available from the Internet by anonymous ftp from zippy.nimh.nih.gov or on floppy disk from NTIS 5285 Port Royal Road, Springfield VA 22161, part number PB93-504868), as done previously. ${ }^{20-23,25}$ For each subject, the first proximal slice not containing gluteal muscle and the next 7-9 distal slices were analyzed. This portion of the thigh was examined because maximal CSA of all of the individual $m$. quadriceps femoris muscles is represented. ${ }^{29}$ A region of interest (ROI) was defined by tracing the outline of $m$. quadriceps femoris. $\mathrm{A} \mathrm{T}_{2}$ for each pixel within the ROI was subsequently determined from the native iamges. Pixels with a $T_{2}$ between $20 \mathrm{~ms}$ and $35 \mathrm{~ms}$ were assumed to represent muscle at rest in the pre-ES images. After spatial calibration, the CSA of these pixels represented $m$. quadriceps femoris CSA. The extent and territory of stimulation was assessed as reflected by pixels with an elevated $\mathrm{T}_{2}$ after ES. The mean and SD of the $T_{2}$ of pixels in each pre ES image were calculated. Pixels in matching post ES images with a $T_{2}>$ the mean plus $1 \mathrm{SD}$ of the $\mathrm{T}_{2}$ of muscle pre ES were considered elevated or to show a contrast shift. The CSA of such pixels was determined. Values were averaged over slices to determine the average absolute and relative CSA of muscle that was stimulated.

MR imaging commenced within 3 min of completion of the third set of each bout of ES. Able-bodied subjects hopped to the imager without bearing weight on the stimulated leg while SCI subjects were transferred maximally with a two person lift. Ablebodied subjects rested 45 min between bouts of ES to allow sufficient recovery of the contraction induced shift in signal intensity, which has a half-life of 57 min. $^{16}$

\section{Statistical analyses}

Statistical analyses were run using the SuperANOVA statistical package (Abacus Concepts, Inc, Berkeley, CA, USA). Descriptive data and $m$. quadriceps femoris CSA were compared between groups using an independent $t$-test. To assess torque evoked by ES independent of fatigue, the average of the first two isometric contractions of set one was calculated. These values and the relative CSA of stimulated muscle were analyzed with a two-way analyses of variance (group by level of ES) with repeated measures over ES level. These torque data were also used to assess the relation between the CSA of stimulated muscle and resultant torque which was done with simple linear regression. The relative decline in torque for each set (1st minus 10th value/1st value times 100 ) and over sets (the aforementioned value for set one, two and three) was compared between groups using a three-way analyses of variance (group by level of ES by set) with repeated measures over ES level and set. Fatigue over a level of ES (1st minus 30th value/1st value times 100) was compared between groups using a twoway analysis of variance (group by ES level) with repeated measures over ES level.

\section{Results}

The SCI and able-bodied adults were comparable regarding age, heights and weight (Table 1). SCI adults had a mean Ashworth spasticity scale of about two and were almost 4 years post injury. The average muscle CSA for $m$. quadriceps femoris was smaller for SCI than able-bodied adults $\left(24 \pm 3\right.$ vs $73 \pm 5 \mathrm{~cm}^{2}$, $P=0.0001)$.

Initial torque for able-bodied adults was quite close to the intended values of $30 \%$ and $60 \%$ of maximal voluntary for mid and high level ES, respectively (Table 2). There was group by level of ES interaction $(P=0.0002)$ concerning initial torque due to a higher

Table 1 Descriptive characteristics of subject

\begin{tabular}{lcc}
\hline Variable & Able-bodied & $S C I$ \\
\hline Age (year) & $27 \pm 3(22-42)$ & $32 \pm 2(22-40)$ \\
Height (cm) & $173 \pm 3(165-185)$ & $169 \pm 3(160-180)$ \\
Weight (kg) & $73 \pm 4(61-90)$ & $71 \pm 4(57-90)$ \\
MVIT (Nm) & $238 \pm 18(128-310)$ & \\
SCI level & & T6 (C6-T12) \\
Ashworth Spasticity & \\
$\quad$ Scale & $1.9 \pm 0.3(0-3)$ \\
Post injury (year) & $4 \pm 1(0.5-12)$ \\
\hline
\end{tabular}

Values are mean \pm SE (range) except for SCI level which is median (range). SCI, spinal cord injury. MVIT, maximal voluntary isometric torque. $n=8$ for each group 
value for able-bodied adults for mid level ES (Table $2)$. The relative decline in torque for a set showed group by set $(P=0.0010)$ and group by level of $\mathrm{ES}$ interaction $(P=0.0256)$ due to the lesser decline shown

Table $2 M$. quadriceps activation and isometric torque evoked by surface electrical stimulation (ES)

\begin{tabular}{lcccc}
\hline Group & Level & $\begin{array}{c}\text { Torque } \\
(\mathrm{Nm})\end{array}$ & CSA (\%) & MVIT (\%) \\
\hline \multirow{3}{*}{ SCI } & low & $30 \pm 3$ & $61 \pm 12$ & \\
& & & & \\
Able-bodied & mid & $44 \pm 6$ & $92 \pm 6$ & \\
& mid & $22 \pm 2$ & $6 \pm 2$ & $9 \pm 2$ \\
& high & $140 \pm 12$ & $38 \pm 4$ & $59 \pm 4$ \\
\hline
\end{tabular}

Values are mean $\pm \mathrm{SE}, n=8$. SCI, spinal cord injury. Level, low, mid and high, three sets of ten isometric actions with ES current set to that which would evoke knee extension with $2.3 \mathrm{~kg}$ attached to the ankle (low), and $30 \%$ (mid) or $60 \%$ (high) of maximal voluntary isometric torque (MVIT) of able-bodied adults. Torque, initial torque evoked by ES. CSA $(\%)$, average CSA of $m$. quadriceps femoris that was stimulated as reflected by an elevated $\mathrm{T}_{2}$. There was a group by level of ES interaction $(P=0.0002)$ concerning initial torque due to a higher value for able-bodied adults for mid level ES. The relative CSA of $m$. quadriceps femoris that was stimulated was greater $(\mathrm{P}=0.0002)$ for low and mid level $\mathrm{ES}$ in SCI as compared to able-bodied adults by able-bodied adults for sets one to three and low and mid level ES (Figure 1). Fatigue for a given level of ES was also less for able-bodied adults $(P=0.0019)$ (Figure 1).

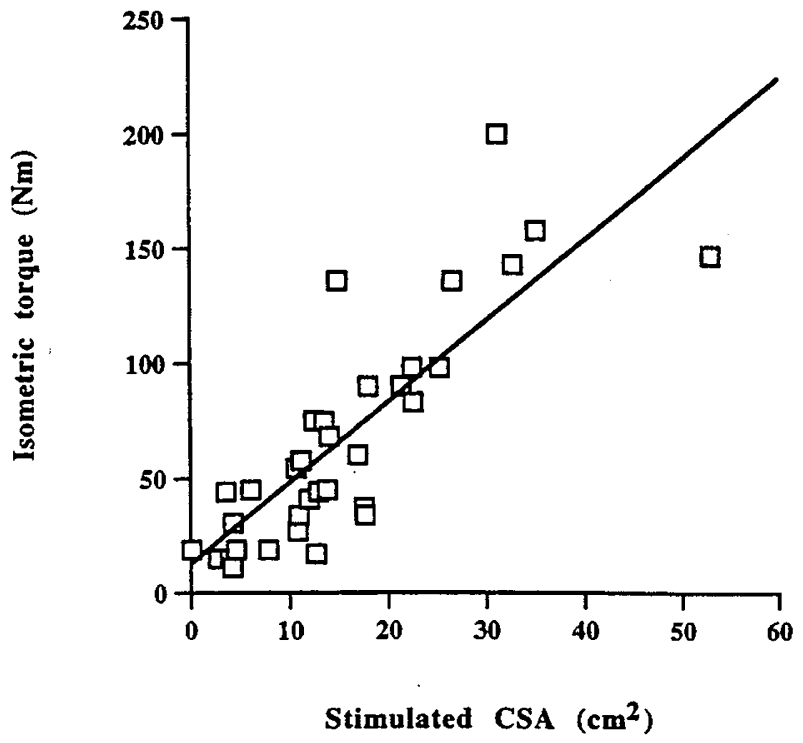

Figure 2 The average cross-sectional area (CSA) of $m$. quadriceps femoris that was activated by surface electrical stimulation plotted versus the resulting torque. Overall, these variables were related, $\mathrm{Nm}=3.53 \times$ stimulated $\mathrm{CSA}+13$, $r^{2}=0.68, P=0.0010$. Symbols represent individual values

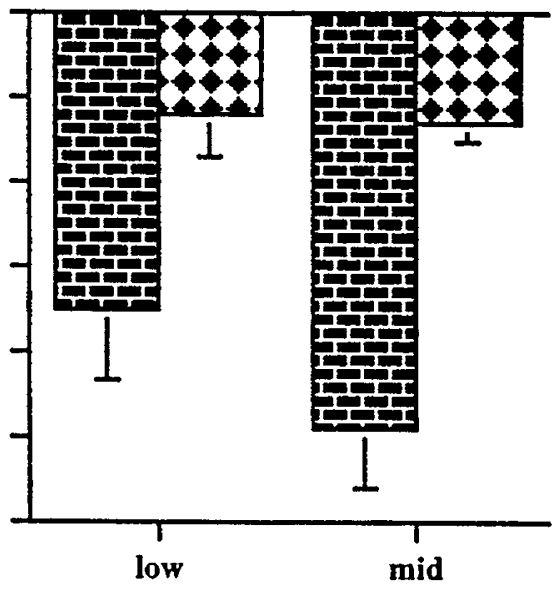

Set

Level

Figure 1 The relative decline in torque for each of three sets (left) or over the three sets (right) of isometric actions evoked by surface electrical stimulations (ES) of $m$. quadriceps femoris. ES current was set to evoke torque sufficient to elicit knee extension with $2.3 \mathrm{~kg}$ attached to the ankle (low level ES) or $30 \%$ of maximal voluntary isometric torque (mid level ES). Each set consisted of $101 \mathrm{~s}$ isometric actions delivered with a $1 / 1$ duty cycle. The percentage decrease in torque showed group by set $(P=0.0010)$ and group by level of ES interaction $(P=0.0256)$ due to the lesser decline shown by able-bodied adults for sets one to three and ES levels low and mid (left). Fatigue over three sets was also less $(P=0.0019)$ for able-bodied adults (right). Values are mean $\pm \mathrm{SE}$ for $n=8$. Left, circle and triangle, able-bodied for low and mid level ES, respectively; square and diamond, corresponding symbols for SCI. Right, bricks are SCI for low and level mid level ES 

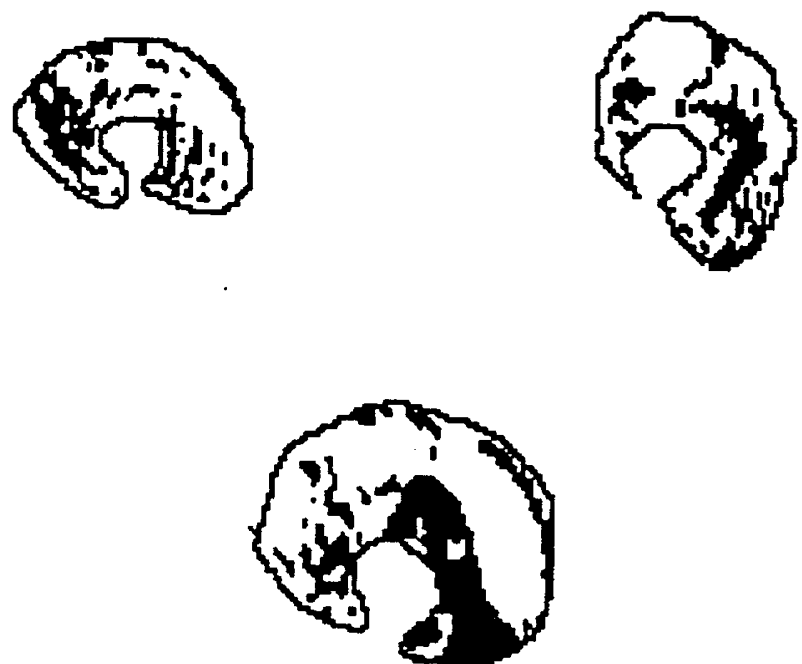

Figure 3 Representative binary $T_{2}$ map of native MRI cross-section of $m$. quadriceps femoris for two different SCI adults (upper) and an able-bodied adult (lower), respectively, showing the territory of stimulation of $m$. quadriceps femoris after mid (upper) and high (lower) level ES. White depicts stimulated muscle, as reflected by elevated pixel $T_{2}$

The relative CSA of $m$. quadriceps femoris that was stimulated was greater $(P=0.0002)$ for low and mid level ES in SCI as compared to able-bodied adults (Table 2). Overall, the average CSA of $m$. quadriceps femoris that was stimulated and the resultant torque, expressed as initial torque for set one, were related (Figure 2). The territory of stimulation of $m$. quadriceps femoris differed among SCI and ablebodied adults even though electrode placement was standardized among subjects (Figure 3).

\section{Discussion}

Surface electrical stimulation (ES) is often used for conditioning skeletal muscle in SCI individuals and as a means for providing assisted standing and walking. ${ }^{30}$ We have previously shown that exercise-induced contrast shift in MR images could be used to map the extent and territory of skeletal muscle activation arising from surface ES in able-bodied individuals in vivo. ${ }^{25}$ It seemed logical to apply this technique to study muscle stimulation in SCI patients to address several important issues. For example, to what extent is atrophy consequent to SCI responsible for the force arising from surface ES? Stimulation of a small portion of the affected muscle could explain the limited force often reported! Alternatively, stimulation of a large portion of an atrophied muscle would suggest that activation per se would not limit the efficacy of ES. If activation is not limiting, what is the contribution of muscle size and fatiguability to force?

The results obtained in this study are similar to those reported previously. ${ }^{25}$ In this and our recent study, able-bodied subjects showed a $10 \%$ to $15 \%$ decline in torque within each set of ten $1 \mathrm{~s}$ isometric actions and complete recovery between sets. Thus, their decline in torque for a set and over three sets were the same (approximately 15\%). The results of both studies also showed that the average CSA of $m$. quadriceps femoris that was stimulated and the resultant torque were related: torque $(\mathrm{Nm})=$ $4.87 \times \mathrm{CSA}+23, \quad r^{2}=0.74, \quad P<0.0001^{25}$ and torque $(\mathrm{Nm})=3.53 \times$ stimulated $\quad \mathrm{CSA}+13, \quad r^{2}=0.68$ $P=0.0010$ (present study). The shorter pulse duration and greater lever arm angle for torque measurement used in this study were probably responsible for the lesser slope of the relation. ${ }^{11,31}$ Taken together, these results lend credence to the use of surface ES and contraction-induced contrast shift in MR images for examining the extent of skeletal muscle activation and contractility arising from electromyostimulation.

The major interest of this study was to assess the influence of SCI on skeletal muscle activation and torque arising from surface ES. This was accomplished using a cross-sectional design in which responses between able-bodied and SCI adults were compared. Both groups showed an increase in torque from low to mid level ES, but the magnitude of this response was greater for able-bodied adults even though the intent was to evoke similar torque in both groups. What limited torque in the SCI adults? Insufficient current from the stimulator is unlikely. Torque often plateaued in spite of increases in current in patients. In adequate stimulation of skeletal muscle is also unlikely. The results suggest ES stimulated $90 \%$ or more of $m$. quadriceps femoris in the SCI adults during mid level ES. Alternatively, the extent of skeletal muscle activation could have been over estimated, and failure of excitation contraction coupling could have limited torque. These explanations are also doubtful. If skeletal muscle activation had been over-estimated, this would suggest that the force generating capacity of skeletal muscle would increase after SCI. The results of spinal transection studies of cats suggest that the specific tension of skeletal muscle is not altered by SCI. ${ }^{32}$ Likewise, Mwave amplitude is maintained during surface ES years after SCI. ${ }^{13,33}$ Accordingly, it seems that atrophy was mainly responsible for the low force we could evoke in patients.

We also examined muscle activation and torque during ES with current that could evoke knee extension with $2.3 \mathrm{~kg}$ attached to the ankle (low level ES). The ability to perform such a weighted knee extension is often used to establish readiness for FES. $^{26}$ When isometric actions were evoked with current sufficient to elicit knee extension with $2.3 \mathrm{~kg}$ attached to the ankle, approximately $60 \%$ of $\mathrm{m}$. quadriceps femoris was stimulated in SCI patients, resulting in a force of about $30 \mathrm{Nm}$. Surface ES in able-bodied adults with the same intent, in contrast, stimulated $6 \%$ of the muscle and evoked a torque of $22 \mathrm{Nm}$. Based on these results, it appears that the 
marked atrophy evident years after SCI necessitates stimulation of more than half of $m$. quadriceps femoris to demonstrate readiness for FES. These results, when combined with the aforementioned discussion of mid level ES, indicate that surface ES can stimulate a large portion of $m$. quadriceps femoris in SCI adults years after injury. The territory of activation may vary among subjects with similar electrode placement, however, depending on ES current. Thus, assuming stimulation of a muscle or a portion of it should be done with caution. Likewise, analyses of biopsy samples to assess biochemical or morphological responses to surface ES should be interpreted with the understanding that all the fibers within the sample may not have been stimulated.

Besides atrophy, increased fatiguability would also be expected to contribute to the limited ability of surface ES to evoke force after SCI. ${ }^{12-14}$ Ten $1 \mathrm{~s}$ strains of $50 \mathrm{~Hz}$ biphasic pulses delivered with a $1: 1$ duty cycle resulted in a decline in force of $-20 \%$ to $-30 \%$ for the SCI patients in this study. Repeating the set of ten isometric actions twice with $1.5 \mathrm{~min}$ of rest between sets resulted in even greater loss of force, $-30 \%$ to $-40 \%$. In either case, the fatigue was greater for SCI than able-bodied adults, as has been noted by others. ${ }^{10-14}$ Conversion to a faster muscle with low mitochondrial content, which would increase energy demand of contraction while lowering capacity for energy supply, have each been suggested to account for the greater fatiguability after SCI. ${ }^{1,4-34}$

The ability to maintain force over a set, contraction one to ten, and over three sets, contraction one to 30 , was the same in able-bodied adults. Thus, they fully recovered between sets. In contrast, SCI adults showed greater fatigue over three sets than over a set, suggesting that their muscle had not fully recovered between sets. This might also be related to a reduced mitochondrial content in affected skeletal muscle after SCI because we have shown that the extent of recovery after intense contractions is directly related to the capacity for aerobic energy supply. ${ }^{35}$ The incomplete recovery of torque between sets might also reflect contraction induced muscle injury which would directly limit force. SCI subjects showed little or no recovery of torque almost $1 \mathrm{~h}$ after low level ES, thus their mid level ES was conducted 2-4 weeks later. If energy imbalance was mainly responsible for their fatigue, it is unlikely that there would be little or no recovery in $60 \mathrm{~min} .{ }^{36}$ Disuse increases susceptibility to contraction induced muscle injury to the extent that high force eccentric muscle actions are not necessary to evoke myofibrillar disruption. ${ }^{37,38}$ This may explain the elevated plasma creatine kinase (CK) activity recently reported for SCI patients after a bout of FES. ${ }^{39}$ While plasma CK is not a direct marker of muscle cell injury, its elevation and the prolonged recovery of force after surface ES found in this study suggest that the contribution of contraction induced fiber disruption to 'fatigue' after SCI needs to be examined.
In summary, the results of this study suggest that atrophy and fatigue are responsible for the limited force that can be evoked by surface ES of skeletal muscle years after SCI. Even though $90 \%$ or more of the affected $m$. quadriceps femoris could be activated, force was a fifth or less of maximal voluntary of ablebodied adults. The reduction in force over repeat contractions, which was as much as $50 \%$, also contributed to the low force evoked by surface ES in patients. This was additive to the large decrement in force attributed to the smaller muscle size evident years after injury.

\section{Acknowledgements}

We thank the subjects, especially the spinal cord injured individuals, for their participation. We also thank St Mary's Hospital, Athens, GA, USA, for assistance in acquiring the MR images. This research was supported by a Doctoral Research Award (95D-16-HIL) from the Foundation for Physical Therapy (EAH), and by Unipatch, Inc, Wabasha, MN, USA, who donated electrodes for electrical stimulation.

\section{References}

1 Round JM, Barr FM, Foffat B, Jones DA. Fibre areas and histochemical fibre types in quadriceps muscle of paraplegic subjects. J Neurol Sci 1993; 116: 207-211.

2 Pacy PJ, Evans RH, Halliday D. Effect of anaerobic and aerobic exercise promoted by computer regulated functional electrical stimulation (FES) on muscle size, strength and histology in paraplegic males. Prosthetics Orthotics Intl 1987; 11: 75-79.

3 Greve JM et al. Functional electrical stimulation (FES): muscle histochemical analysis. Paraplegia 1993; 31: $764-770$.

4 Martin TP, Stein RB, Hoeppner PH, Reid DC. Influence of electrical stimulation on the morphological and metabolic properties of paralyzed muscle. J Appl Physiol 1992; 72: 1401 1406.

5 Scelsi R et al. Muscle fiber type morphology and distribution in paraplegic patients with traumatic cord lesion. Acta Neuropathol 1982; 57: $243-248$

6 Andersen JL et al. Myosin heavy chain isoform transformation in single fibres from $\mathrm{m}$. vastus lateralis in spinal cord injured individuals: effects of long-term functional electrical stimulation (FES). Pflügers Archiv 1996; 431: 513-518.

7 Grimby G, Broberg C, Krotkiewska, Krokiewski M. Muscle fiber composition in patients with traumatic cord lesion. Scand $J$ Rehab Med 1976; 8: $37-42$.

8 Burnham $\mathrm{R}$ et al. Skeletal muscle fibre type transformation following spinal cord injury. Spinal Cord 1997; 35: 86-91.

9 Rochester L et al. Influence of electrical stimulation of the tibialis anterior muscle in paraplegic subjects. 2. Morphological and histochemical properties. Paraplegia 1995; 33: 514-522.

10 Rabischong E, Ohanna F. Effects of functional electrical stimulation on evoked muscular output in paraplegic quadriceps muscle. Paraplegia 1992; 30: 467-473.

11 Levy M, Mizrahi J, Susak Z. Recruitment, force and fatigue characteristics of quadriceps muscles of paraplegics isometrically activated by surface functional electrical stimulation. $J$ Biomed Eng 1990; 12: $150-155$.

12 Rochester L et al. Influence of electrical stimulation of the tibialis anterior muscle in paraplegic subjects. 1. Contractile properties. Paraplegia 1995; 33: $437-449$.

13 Stein RB et al. Optimal stimulation of paralyzed muscle in spinal cord injured patients. J Appl Physiol 1992; 72: 1393-1400. 
14 Shields RK. Fatiguability, relaxation properties, and electromyographic responses of the human paralyzed soleus muscle. $J$ Neurophysiol 1995; 73: 2195-2206.

15 Adams GR, Duvoisin MR, Dudley GA. Magnetic resonance imaging and electromyography as indexes of muscle function. $J$ Appl Physiol 1992; 73: $1578-1583$.

16 Fisher MJ et al. Direct relationship between proton T2 and exercise intensity in skeletal muscle MR images. Invest Radiol 1990; 25: $480-485$

17 Fleckenstein JL, Canby RC, Parkey RW, Peshock RM. Acute effects of exercise on MRI of skeletal muscle in normal volunteers. Am J Roentgenol 1988; 151: 231 - 237.

18 Fleckenstein JL et al. Exercise enhanced MR imaging in variations in forearm muscle anatomy and use: importance in MR spectroscopy. Am J Roentgenol 1989; 153: 693-698.

19 Jenner $\mathrm{G}$ et al. Changes in magnetic resonance images of muscle depend on exercise intensity and duration, not work. $J$ Appl Physiol 1994; 76: $2119-2124$

20 Ploutz LL, Tesch PA, Biro RL, Dudley GA. Effect of resistance training on muscle use during exercise. J Appl Physiol 1994; 76: $1675-1681$

21 Ploutz-Snyder LL, Tesch PA, Crittenden DJ, Dudley GA. Effect of unweighting on skeletal muscle use during exercise. $J$ Appl Physiol 1995; 79: $168-175$.

22 Conley MS et al. Non-invasive analysis of human neck muscle function. Spine 1995; 20: 2505-2512.

23 Conley MS, Stone MH, Nimmons M, Dudley GA. Resistance training and human cervical muscle recruitment plasticity. $J$ Appl Physiol 1997; 83: 2105-2111.

24 Yue G et al. Sensitivity of muscle proton spin-spin relaxation time as an index of muscle activation. J Appl Physiol 1994; 77: $84-92$.

25 Adams GR, Harris RT, Woodard D, Dudley GA. Mapping of electrical muscle stimulation using MRI. J Appl Physiol 1993; 74: $532-537$.

26 Arnold PB et al. FES: Its efficacy and safety in improving pulmonary function and musculoskeletal fitness. Arch Phys Med Rehab 1992; 73: $665-668$.
27 Conley MS et al. Effect of acute head-down tilt on skeletal muscle cross-sectional area and proton transverse relaxation time. $J \mathrm{Appl}$ Physiol 1996; 81: $1572-1577$.

28 Hather BM, Adams GA, Tesch PA, Dudley GA. Skeletal muscle responses to unilateral lower limb suspension. J Appl Physiol 1992; 72: 1493 - 1498 .

29 Narici MV et al. Changes in force, cross-sectional area and neural activation during strength training and detraining of the human quadriceps. Eur J Appl Physiol 1989; 59: 310-319.

30 Gordon T, Mao J. Muscle atrophy and procedures for training after spinal cord injury. Phys Therapy 1994; 74: 50-60.

31 Hultman E, Sjoholm H, Jaderholm-Ek I, Krynicki J. Evaluation of methods for the electrical stimulation of human skeletal muscle in situ. Pflügers Archiv 1983; 398: 138-141.

32 Roy RR, Baldwin KM, Edgerton VR. The plasticity of skeletal muscle: effects of neuromuscular activity. Exerc Sport Sci Rev 1991; 19: 269 - 312 .

33 Shields RK. Fatiguability, relaxation properties, and electromyographic responses of the human paralyzed soleus muscle. $J$ Neurophysiol 1995; 73: 2195 - 2206.

34 Crow MT, Kushmerick MJ. Chemical energetics of slow- and fast-twitch muscles of the mouse. J Gen Physiol 1982; 79: 147 166.

35 Jansson E, Dudley GA, Norman B, Tesch PA. Metabolic and force recovery after high intensity exercise in relation to the oxidative potential of skeletal muscle. Acta Physiol Scand 1990; 139: $147-152$

36 Fitts RH. Cellular mechanisms of muscle fatigue. Physiol Rev 1994; 71: $49-94$

37 Ploutz-Snyder LL, Tesch PA, Hather BA, Dudley GA. Vulnerability to dysfunction and muscle injury after unloading. Arch Phys Med Rehab 1996; 77: 773- 777.

38 Warren GL et al. Eccentric contraction-induced injury in normal and hindlimb-suspended mouse soleus and EDL muscles. J Appl Physiol 1994; 77: 1421 - 1430.

39 Robergs RA et al. Increased endothelin and creatine kinase after electrical stimulation of paraplegic muscle. J Appl Physiol 1993; 75: $2400-2405$. 